\title{
Use of orthogonal or parallel plating techniques to treat distal humerus fractures.
}

\author{
Joshua M. Abzug \\ Thomas Jefferson University \\ Phani K. Dantuluri \\ Emory University Midtown Hospital
}

Follow this and additional works at: https://jdc.jefferson.edu/orthofp

Part of the Orthopedics Commons

Let us know how access to this document benefits you

\section{Recommended Citation}

Abzug, Joshua M. and Dantuluri, Phani K., "Use of orthogonal or parallel plating techniques to treat distal humerus fractures." (2010). Department of Orthopaedic Surgery Faculty Papers.

Paper 26.

https://jdc.jefferson.edu/orthofp/26

This Article is brought to you for free and open access by the Jefferson Digital Commons. The Jefferson Digital Commons is a service of Thomas Jefferson University's Center for Teaching and Learning (CTL). The Commons is a showcase for Jefferson books and journals, peer-reviewed scholarly publications, unique historical collections from the University archives, and teaching tools. The Jefferson Digital Commons allows researchers and interested readers anywhere in the world to learn about and keep up to date with Jefferson scholarship. This article has been accepted for inclusion in Department of Orthopaedic Surgery Faculty Papers by an authorized administrator of the Jefferson Digital Commons. For more information, please contact: JeffersonDigitalCommons@jefferson.edu. 


\title{
As submitted to:
}

\author{
Hand Clinics
}

\author{
And later published as:
}

"Use of Orthogonal or Parallel Plating Techniques to

\author{
Treat Distal Humerus Fractures"
}

Volume 26, Issue 3, August 2010, Pages 411-421

doi:10.1016/j.hcl.2010.05.008

Joshua M. Abzug, MD and Phani Dantuluri, MD

Dr. Abzug is a hand surgery fellow, Thomas Jefferson University Hospital, The Philadelphia Hand Center, Philadelphia, PA

Dr. Dantuluri is Department of Orthopaedics, Emory University Midtown Hospital. 


\section{Synopsis:}

Distal humerus fractures continue to be a complex fracture for the surgeon to treat. This article has described two techniques that can be utilized to tackle these difficult fractures. Both of these techniques have yielded excellent outcomes after ORIF, however, both techniques also have significant complications associated with them. Use of parallel plating or orthogonal plating will be depend on surgeon preference and the fracture pattern present. The key to successful treatment of these fractures is obtaining anatomic reduction with stable fixation to allow early range of motion. 


\section{Introduction:}

Fractures of the distal humerus have been shown to account for $2-6 \%$ of all fractures. (1) These fractures occur in a bimodal age distribution, with fractures in younger patients occurring as a result of high energy mechanisms and fragility fractures occurring in the elderly as a result of low energy falls. (2) The subsequent fracture pattern present may be extra-articular ( $A O$ type $A$ ), partial articular (AO type B), or complete articular (AO type C.) (3) Other classification systems utilized are the Jupiter and Mehne (4) system which is based on fracture patterns observed intraoperatively and the system proposed by Davies and Stanley which combines the aforementioned classifications into one system. (5) Whatever system is utilized, it is important to pay particular attention to the mechanism of injury, the condition of the soft tissues, the bone quality, and lastly the age and physical demands of the patient.

All of these fractures represent a challenge to the surgeon due to the distal location and predilection towards articular involvement. Due to these issues multiple treatment strategies have emerged with the majority of current recommendations including open reduction and internal fixation (ORIF.) ORIF of the fracture allows the surgeon to restore anatomical alignment of the fracture fragments and permit early range of motion exercises which may aid in the return of a functional range of motion of the elbow postoperatively. Different methods of internal fixation of the fracture fragments have evolved over time in 
an attempt to best restore anatomical alignment of the distal humerus, given its complex anatomy while also providing stable fixation to permit early rehabilitation of the injured extremity.

The distal humerus is composed of a medial and lateral column with a central area of thin weaker bone. This central area, the coronoid and olecranon fossae, is present to facilitate elbow flexion and extension by allowing a space for the olecranon tip to articulate, while also providing bony stability. However, this central area has been shown to be particularly thin in patients with osteopenia thus making it a common site of involvement with fractures of the distal humerus. (6) It is essential that this area be reconstructed to restore diaphyseal-metaphyseal contact in order to provide the most stability and allow for the best healing potential. (7) Due to this unique anatomy of the distal humerus, various plates have been developed to try and provide adequate stability to the articular, metaphyseal, and diaphyseal regions of the distal humerus. These plates include Y-shaped plates, recon plates contoured to the anatomy, and recently, precountoured plates with or without locking screw capabilities.

The anatomical location to place the plates on the distal humerus has recently been debated throughout the literature with the majority of authors currently recommending at least two plates be utilized to provide adequate stability and allow for adequate restoration of anatomy. Orthogonal plating, 
otherwise known as $90-90$ plating or perpendicular plating, involves placing one plate on the medial column of the distal humerus and the other plate along the posterolateral column. The concept of parallel plating involves placing one plate along the medial column of the distal humerus and the other plate along the lateral column.

\section{Anatomy:}

The elbow joint is characterized as being a hinge joint since it only has a single axis of rotation. (8) This rotation primarily occurs between the semilunar notch at the proximal part of the ulna and the trochlea at the distal end of the humerus. The trochlea is bounded on each side by a bony column, thus forming a bony construct that is analogous to a trianglelin. If any of the arms of the triangle are disrupted, the entire construct is weakened more than expected.

(9) Therefore, it is important to ensure each arm of this bony construct has adequate fixation when performing an ORIF of the distal humerus.

The medial and lateral bony columns surrounding the trochlea have different anatomic extensions. The medial column terminates approximately one centimeter proximal to the distal end of the trochlea, whereas the lateral column extends to the distal aspect of the trochlea. (10) The anterior surface at the distal extent of lateral column is covered with articular cartilage, thus forming the capitellum. 
On the anterior aspect of the distal humerus the coronoid fossa is present just proximal to the trochlea and the radial fossa is present just proximal to the capitellum. These fossae are separated by a longitudinal bony ridge that continues distally with the lateral lip of the trochlea. (10) The longitudinal ridge and the lateral lip of the trochlea form the anterior anatomic division between the medial and lateral columns.

On the posterior aspect of the distal humerus the olecranon fossa is present to accommodate the tip of the olecranon when the elbow is in full extension. The distal humerus itself is quite thin between the medial and lateral columns at this level because the intramedullary canal actually tapers to an end approximately two to three centimeters proximal to the olecranon fossa. In fact, six percent of the population may have an actual bony defect, a septal aperture, in this area. (10)

The medial column of the distal humerus diverges from the humeral shaft at approximately a 45 degree angle. The proximal two thirds of this column is made up of cortical bone, while the distal third is formed by the medial epicondyle, composed of cancellous bone. (11) The lateral column of the distal humerus is subtended at approximately a twenty degree angle in reference to the humeral shaft. The proximal half of the lateral column is composed of cortical bone, while the distal half is composed of cancellous bone. (10) The proximal portion not only is composed of cortical bone, but also 
is a flat and broad surface, thus making it ideal for placement of a plate. (Figure 1)

\section{Classification Schemes:}

Fractures of the distal humerus have historically been classified based on anatomical considerations. Initially these fractures were classified based on the concept that the distal end of the humerus was made up of condyles. The terms supracondylar, condylar, transcondylar, and bicondylar fractures were utilized. (12)

Currently, fractures of the distal humerus are more commonly described based on the previously discussed anatomical description of the columnar structure of the distal humerus. This includes describing fractures as single columnar, bicolumnar, and transcolumnar fractures. In addition, fractures can be classified based on the specific bony fragment involved; ie. trochlear, capitellar, medial epicondylar, or lateral epicondylar fractures.

Single column fractures make up 3-4\% of distal humerus fractures and more commonly involve the lateral column. (13) These fractures involve the medial or lateral column and extend distally through the intercolumnar portion of the distal humerus. (10)

Bicolumnar fractures are the most common type of distal humerus fracture representing 5-62\% of distal humerus fractures. (10) These fractures 
involve each limb of the "triangle" (Figure 2) discussed earlier, thus making them extremely difficult to treat. (10) It is this difficulty that has led to the ongoing debate over which type of fracture fixation is best in order to provide the most rigid fixation that will facilitate rapid healing while still allowing early range of motion.

\section{Rationale:}

\section{Orthogonal Plating}

Orthogonal plating techniques evolved after a publication by Jupiter and colleagues in 1985, reporting on patients having successful outcomes with ORIF of distal humerus fractures. (14) This retrospective series looked at 39 patients treated with ORIF of the distal humerus and found 27 patients to have good or excellent results. They noted that the key to surgical success was obtaining enough bony stability to permit early range of motion. This usually required the utilization of two plates, one on the medial column and the other on the lateral column. (14)

Prior to this report, the literature had a wide range of treatment recommendations ranging from non-operative treatment to ORIF with limited internal fixation. These series had few numbers and utilized different outcome measures, thus making comparisons among various treatment methods extremely difficult. (10) Despite this, it became accepted that Kirschner wire fixation alone did not provide adequate stability to treat bicolumnar distal 
humeral fractures. $(15,16)$ In addition, Waddell and colleagues have shown that elbow immobilization of three to four weeks post-operatively leads to unacceptable stiffness. (17) One caveat to this is that if the fracture is severely comminuted and the fixation is suboptimal, it may be better to immobilize the elbow for an extended period to allow fracture union and then deal with a stiff elbow with fracture union as that may be preferable to failure of fixation and a nonunion which may result from an attempt at early mobilization in these patients.

Based on these observations, Jupiter and colleagues established the technique of orthogonal plating to provide adequate stability of the fracture fragments to allow bony healing and early postoperative rehabilitation.

\section{Parallel Plating}

The concept of parallel plating was conceived because some surgeons felt that the described technique of orthogonal plating was not sufficient for all cases as they felt there were some cases where orthogonal plating provided inadequate fixation of the distal fragments and not enough stability between the intraarticular distal fragments and the humeral shaft. Several authors have documented a 20 to 25 percent rate of unsatisfactory result following orthogonal plating of distal humerus fractures. Henley et al. had failure of fixation in five of thirty-three patients, while Letsch et al. had failures in five of 
eighty eight fractures. $(18,19)$ There were failures in three of fifty-seven patients treated by Holdsworth and Mossad, while Wildburger's series demonstrated failure in nine of seventy-two fractures. (20,21) Additionally, Sodergard et al. had failures in sixteen of ninety-six fractures. (22) When fixation does fail, it occurs at the supracondylar level. (23) This occurs due to suboptimal anchorage of the articular fragments to the shaft due to the limited number and length of screws that can be placed in the distal fragments. (23)

When early motion is permitted in fractures treated without adequate stability, motion occurring at the fracture site can lead to nonunion. (24) Korner et al. noted that seventy five percent of malunion or nonunion cases were caused by inadequate initial fracture fixation. (25) Alternatively, if the elbow was immobilized for a prolonged period of time to accommodate for the tenuous fixation, resultant elbow stiffness may occur. (26) An additional rationale for utilization of parallel plating is that longer screws can be placed from a medial to lateral direction as opposed to a screw placed through a posterolateral plate. (6) Based on these observations, the Mayo clinic group proposed the idea of parallel plating utilizing the principles of enhancing fixation of the distal fragments and achieving stability at the supracondylar level. (23) There are eight technical objectives that have been described concerning parallel plating. Six of these objectives are related to distal screw insertion and two are related to plate fixation. 
With regard to distal screw insertion, each screw should pass through a plate. Additionally, each screw should engage a fragment on the opposite side that is also fixed to a plate. There should be an adequate number of screws placed in the distal fragments and each screw should be as long as possible engaging as many articular fragments as possible. Lastly, the screws should lock together by interdigitation, creating a "fixed-angle" structure and linking the columns together. (27) With regard to fixation of the plates, the plates should be applied allowing for compression of both columns at the supracondylar level. Lastly, the plates chosen must have enough strength and stiffness to resist breaking or bending before union occurs at the supracondylar level. (27) It was the Mayo clinic group's belief that following these technical objectives would allow the parallel plating technique to link both columns of the distal humerus, thus providing the structural stability necessary for fracture healing. The interdigitation of the distal screws is likened to a keystone of an arch, being the structural link necessary for adequate fixation. (27) Thus, fixation of the bone fragments relies on the stability of the hardware construct rather than on screw purchase in the bone. (23)

\section{Biomechanical Anaylsis:}

The literature has had contradictory results with regard to biomechanical testing of these two techniques. Self and colleagues assessed the biomechanical aspects of the two techniques utilizing reconstruction plates and 
found the parallel system to be stronger and stiffer. (28) Jacobson and colleagues also assessed the biomechanical aspects of the techniques with reconstruction plates yet found the perpendicular system to be stronger. (29) Both of these studies were performed on cadaveric bone.

Schwartz and colleagues assessed nonlocking periarticular plates on composite bone and found similar biomechanical properties in both techniques. (30) More recently, Stoffel and colleagues assessed the biomechanical principles of the techniques utilizing locking plates. The authors utilized 24 humeri from fresh-frozen female cadavers and found stability was most dependent on bone quality. However, within their analysis the parallel plating system was found to have significantly higher stability in compression and external rotation, as well as a greater ability to resist axial plastic deformation. (31)

Schuster et al. also utilized cadaveric bones to assess the biomechanical properties of various plates utilizing the orthogonal plating technique for simulated type C2 distal humerus fractures. Their assessment included a comparison of fractures treated with conventional reconstruction plates, locking compression plates, and locking distal humerus plates. Similar to the aforementioned study by Stoffel et al., this study determined that stability was dependent on bone mineral density. When good bone quality was present, the choice of implant did not matter. However, when bone mineral density was 
low, less than $420 \mathrm{mg} / \mathrm{cm}^{3}$, both locking plates provided superior resistance against screw loosening compared to the non-locking conventional reconstruction plate. (32) Based on this biomechanical data the authors recommend utilization of locking plates for communited and/or osteopenic fractures.

\section{Surgical Techniques:}

ORIF should be performed in fractures with any amount of significant displacement involving the articular surface as outcomes are superior to nonsurgical treatment of these fractures. The goals of ORIF include restoration of the elbow joint anatomy with stable fixation to permit early motion.

\section{Orthogonal Plating}

The technique for orthogonal plating is the technique originally described and recommended by the AO group. (see Figures 5-12, courtesy of Dr Scott Steinmann) The patient is placed either supine with the affected extremity draped across the patient's chest or in the lateral decubitus position. A midline posterior skin incision is utilized with or without a slight curvature medial or lateral to the olecranon to avoid incising directly over it. It is imperative that the ulnar nerve be identified and mobilized to avoid damage to this structure. Gofton et al. recommended mobilizing the ulnar nerve distally to the first motor branch of the flexor carpi ulnaris. Subsequently, these authors release the cubital tunnel retinaculum as well as the aponeurosis between the humeral and ulnar origins of 
the flexor carpi ulnaris. Proximally the intermuscular septum and Arcade of Struthers are resected. The ulnar nerve is then transposed anteriorly, with the intention to later perform a formal anterior subcutaneous transposition. (33) Other authors feel it is unnecessary to transpose the ulnar nerve, but it does need to be mobilized enough to permit access to the distal humerus without the nerve being injured.

Once the ulnar nerve is mobilized the distal humerus is approached through a triceps sparing approach, a triceps splitting approach or an olecranon osteotomy. The triceps splitting and triceps sparing approaches allow visualization of the posterior portion of the trochlea, but only the olecranon osteotomy permits access to the anterior portions of the trochlea and capitellum. (34) The rationale for utilizing a triceps sparing or triceps splitting approach is to avoid the complications of an olecranon osteotomy such as prominent hardware, delayed unions, or nonunions. Despite these complications, the olecranon osteotomy is thought to provide optimal exposure to the intra-articular surface of the distal humerus. In addition, by performing the osteotomy, complications involving the triceps can be avoided. These include disrupting the elbow extensor mechanism, fibrosis of the triceps, and intramuscular nerve injuries. (35) Mckee et al. retrospectively compared patient outcomes between the triceps splitting approach and olecranon osteotomies in a series of open distal humerus fractures as well as in a series of closed distal humerus fractures. $(36,37)$ Patient outcome measures including DASH scores, 
the Mayo elbow score and SF-36 Physical function scores were recorded. The authors of both series reported better outcomes with the triceps splitting approach compared with an olecranon osteotomy. $(36,37)$

The olecranon osteotomy is started with the use of an oscillating saw but it is not completed. An osteotome is utilized to complete the osteotomy. If the distal humeral fracture does not have significant articular segment comminution, a triceps spltting approach to the distal humerus can be performed. This is done by reflecting equal portions of the medial and lateral triceps aponeurosis and detaching them off of the olecranon. Lastly, a triceps sparing approach can be utilized with extra-articular fractures or simple intraarticular fractures by working medial and lateral to the triceps.

Once the fracture fragments are identified and reduced, provisional fixation is performed with Kirschner wires. Care must be taken here to pay attention to neurovascular structures around the elbow as the provisional Kirschner wires can injure these structures if left too long or too sharp. The orthogonal plates are then applied to the bone with the medial one being placed along the medial column of the distal humerus and the second plate being placed along the posterolateral aspect of the lateral column. The fixation should ideally have at least three screws proximal and three screws distal to the fracture site through each plate and thus through each column. When reconstruction plates are utilized, insufficient stability may be present and 
require placing a third reconstruction plate along the lateral aspect of the lateral column. This was necessary in 40 percent of patients in the Gofton et al. series of AO Type C distal humerus fractures. (33)

Once the plates are secured to the distal humerus, the elbow range of motion is assessed to ensure adequate stability is present without a mechanical block. If the triceps splitting approach was performed, the triceps is reattached to the olecranon via non-absorbable suture passed through drill holes in the olecranon. The medial and lateral aspects of the triceps aponeuorsis are subsequently sutured to each other and the remainder of the wound is closed in layers.

If an olecranon osteotomy was performed, multiple techniques are available to provide fixation of the osteotomy site. These include utilizing a tension band technique, intramedullary screw fixation with or without a tension band, or placement of an olecranon plate. If an intramedullary screw is placed, it must be of sufficient size and length to obtain adequate purchase in the proximal ulna. Gofton et al. recommended utilizing a contoured $3.5 \mathrm{~mm}$ reconstruction plate to provide the most reproducible results. Their series had no nonunions and no isolated procedures for hardware removal. (33) Additionally, the authors stated that less moribidity is associated with hardware removal compared to re-operation for an olecranon nonunion. (33) Other authors prefer a stronger plate, such as an LCD plate, with or without locking screws to provide 
additional strength to the construct. Lastly, if one chooses to fix the olecranon osteotomy with a tension band technique, an option is to utilize two 20 gauge wires to create two figure of eight tension bands. This small wire size may obviate the need for hardware removal and avoid other potential hardware related complications.

\section{Parallel Plating}

The technique for parallel plating was described in 2007 by O'Driscoll and colleagues from the Mayo clinic. (23) (see Figure 13-17, courtesy of Dr David Ring) The patient is positioned in the supine position and a sterile tourniquet is applied. Subsuequently a triceps-anconeus reflecting pedicle (TRAP) approach is performed after the ulnar nerve is transposed anteriorly. The goal of the TRAP approach is to reflect the triceps in continuity with the anconeus. The utilization of an olecranon osteotomy is recommended when intra-articular communition is present. (27)

Attention is initially directed at the articular surface of the distal humerus to ensure an adequate reduction. The articular surface is reassembled with smooth Kirschner wires to provisionally hold the reduction in place utilizing the proximal portion of the ulna and radial head as templates if necessary. The Kirschner wires should be placed close to the subchondral bone to ensure that they do not interfere with placement of your screws into the distal fragment. If during the reduction missing bone is encountered, one should understand that 
the anterior aspect of the distal part of the humerus is the critical area that needs to be restored to allow for a functional joint. (27) The posterior aspect of the articular surface of the distal humerus is a less critical region. In addition, the medial half of the trochlea is vital to ensure stability of the elbow articulation. It can be reconstructed with either the lateral half of the trochlea or the capitellum. (27)

Once the articular surface is anatomically reduced, the plates are placed along the medial and lateral columns of the distal humerus. One-third tubular plates are not strong enough for fixation of these fractures and therefore, the precountered distal humerus plates are currently favored. However, if the surgeon is contouring the plates, it should be recognized that it is preferable to undercontour the plates to allow for additional compression at the metaphyseal region when they are applied. The plates should be long enough to allow for at least three screws to be placed in the proximal part of the humeral shaft proximal to the metaphyseal component of the fracture. Additionally, the plates should end at different levels to avoid creating a stress-riser. (27) (Figures $3,4)$

Once the appropriate size plates are chosen, they are held in place by driving a smooth Steinmann pin through the respective epicondyle, medially or laterally. Subsequently, one cortical screw is introduced in the slotted hole of each plate to accommodate for minor adjustments in plate position. Following 
adjustment of plate height, a large bone clamp is utilized to compress the intraarticular fracture fragments, supposing there is no missing bone. This allows for interfragmentary compression of the intra-articular fragments without the need for lag screws. Once the clamp is in place, the distal screws are inserted to secure the intra-articular fragments to the plates. These distal screws should be as long as possible, pass through as many fragments as possible, and engage the opposite column. (27)

Once the distal fragments are secured to the plates, attention is turned to the supracondylar region. One of the screws in the slotted holes is backed out and a large bone clamp is placed to eccentrically load the supracondylar region. This is accomplished by placing the clamp distally on the side the screw was backed out of and proximally on the opposite side. A proximal screw is then inserted in compression mode and the slotted screw is retightened. (27) During this maneuver it is important to ensure that the varus-valgus and rotational alignments were not altered. The same process of loosening the slotted screw, applying the bone clamp, placing a proximal screw, and retightening the slotted screw is now performed on the opposite side. The remaining screws are now placed to allow for additional stability. The holes created by the Steinmann pins in the epicondyles can be utilized as pilot holes for placement of screws into these areas. (27) 
Once the plates are affixed to the humerus, the elbow is taken through a range of motion of flexion-extension as well as pronation-supination to ensure no mechanical blocks are present. One deep and one subcutaneous drain are placed and the wounds are closed. The arm is then placed in a bulky noncompressive dressing with an anterior plaster splint to maintain full extension. The dressing is removed approximately three to five days post-operatively and physical therapy including active and passive motion is begun.

\section{Outcomes:}

\section{Orthogonal Plating}

Gofton et al. performed a retrospective review of 23 patients treated with dual orthogonal plates at a mean follow-up of 45 months. Their results demonstrated that patients had minimal subjective deficits, ten percent, with a mean overall satisfaction score of 93 percent. The mean DASH score at the most recent follow-up was 12 , with a range from 0 to 38 , whereas the mean ASES score was 9.7, plus or minus 10.1 points. The mean amount of flexion achieved was 142 degrees with a mean flexion contracture of 19 degrees. No significant differences in pronation or supination were noted between the affected and unaffected sides. A statistically significant decrease in strength was present in all strength parameters assessed. (33)

Assessment of the post-operative radiographs did not demonstrate any distal humeral articular step-offs or gaps greater than two millimeters. In 
addition, there were no nonunions or malunions indentified in the distal humerus. The amount of post-traumatic arthritis present, as classified by Knirk and Jupiter, was grade 0 or 1 in 18 patients, and grade 2 or 3 in 5 patients. $(33,38)$

\section{Parallel Plating}

Sanchez-Sotelo et al. discussed their retrospective results of thirty-two patients treated with parallel plating over a ten year period when they described the surgical technique. Thirty-one of the thirty-two patients went on to union without requiring additional surgery and none of the patients had failure of their hardware or fracture displacement. (23) The average flexionextension arc at latest follow-up was 99 degrees but five patients did require excision of heterotopic ossification secondary to elbow stiffness. The mean MEPS was 85 points with twenty seven patients having a good or excellent result and five patients having a fair or poor result. (23)

Athwal and colleagues also recently published a retrospective review of AO/OTA type $C$ fractures treated with the Mayo Elbow parallel plating system. In their series of 32 patients, the average flexion-extension arc, at a mean of 27 months follow-up, was 97 degrees. The mean DASH score was 24 points while the mean Mayo Elbow Performance score was 82 points. They noted no implant failures and nonunions. (39)

\section{Complications:}


Complications arise whether utilizing the orthogonal plating technique or the parallel plating technique. These include heterotopic ossification, failure of fixation, nonunion, malunion, infection, ulnar neuropathy and complex regional pain syndrome.

In the orthogonal plating series of 23 patients by Gofton et al. about half of the patients experienced at least one complication, with the presence of heterotopic ossification being the most common, present in thirty percent of patients. These authors reported no loss of fixation and no cases of ulnar neuropathy. An olecranon nonunion was present in two patients, both of which required an additional procedure to achieve union. No distal humeral nonunions or malunions were noted. (33)

In the recently published retrospective series of Athwal et al. assessing the Mayo Elbow parallel plate technique, they noted a complication rate of 53 percent, with complications arising in 17 of 32 patients. The most common complication noted was postoperative nerve injuries, present in five patients, 16 percent, of which three had completely resolved by 4.5 months postoperatively. Four patients, 12 percent, did experience wound complications including two wound dehiscences requiring surgical debridement. One olecranon nonunion was noted which was treated non-operatively. (39)

\section{Nonunion Scenarios:}


Similar to the difficulties encountered treating distal humerus fractures, treatment of distal humeral nonunions has proven to be a difficult entity for the surgeon. This complication is especially devastating when elbow instability is so severe that the limb cannot be supported against gravity. Treatment of this problem can be achieved with total elbow arthroplasty, however many patients are too young or active for total elbow arthroplasty to be the optimal treatment. Therefore, some authors have suggested utilizing multiple plates to provide adequate stability in order to achieve osseus union. (26)

Ring et al. retrospectively reviewed fifteen patients with distal humeral nonunions treated with multiple plates to achieve adequate osseous stability. The orthogonal plating technique, with autogenous bone-grafting, was performed in five patients, while the remaining patients required a third or fourth plate to achieve adequate fixation. Twelve of the fifteen patients achieved union, while the remaining three patients went on to have a total elbow arthroplasty performed. After a minimum of two year follow-up, the average flexion achieved was 117 degrees with a flexion contracture present averaging

22 degrees. Eleven of the twelve patients who achieved union had excellent or good functional results according to the Mayo Elbow Performance Index. (40)

\section{Conclusion:}

Distal humerus fractures continue to be a complex fracture for the surgeon to treat. This article has described two techniques that can be utilized 
to tackle these difficult fractures. Both of these techniques have yielded excellent outcomes after ORIF, however, both techniques have significant complications associated with them. Use of parallel plating or orthogonal plating will be depend on surgeon preference and the fracture pattern present. Orthogonal plating may be preferred in cases of an anterior shear fracture where the fixation from posterior to anterior will provide additional stability to the intra-articular fractures. Parallel plating may be the preferred technique utilized for very distal fracture patterns since more stability can be obtained by providing additional screws in the distal fragment. The key to successful treatment of these fractures is obtaining anatomic reduction with stable fixation to allow early range of motion. Performing anatomic reductions while minimizing soft tissue trauma will lead to improved patient outcomes while minimizing the complication rates. 


\section{References:}

1. Srinivasan K, Agarwal M, Matthews SJE, et al. 2005. Fractures of the distal humerus in the elderly. Clin Orthop Rel Res. 434:222-30.

2. Robinson CM, Hill RMF, Jacobs N, et al. Adult distal humerus metaphyseal fractures: epidemiology and results of treatment. J Orthop Trauma. $2003 ; 17: 38-47$.

3. Muller ME, Allgower M, Schneider R, et al. Manual of internal fixation techniques recommended by the AO group. Ed. 2, New York. Springer; 1979:176-7.

4. Jupiter JB, Mehne DK. Fractures of the distal humerus. Orthopedics. $1992 ; 15: 825-33$.

5. Davies MB, Stanley D. A clinically applicable fracture classification for distal humeral fractures. J Should Elb Surg. 2006;15:602-8.

6. Leugmair M, Timofiev E, Chirpaz-Cerbat JM. Surgical treatment of AO type C distal humerus fractures: internal fixation with a Y-shaped reconstruction (Lambda) plate. J Shoulder Elbow Surg. 2008;17:113-20. 
7. Seth AK, Baratz ME. Fractures of the elbow. In: Trumble TE, Budoff JE, Cornwall R, eds. Hand, elbow, \& shoulder. Philadelphia:Mosby, 2006:52231.

8. London JT. Kinematics of the elbow. J Bone Joint Surg. 1981;63:529-36.

9. Mehne DK, Matta J. Bicolumn fractures of the adult humerus. Paper presented at the 53rd annual meeting of the American Academy of Orthopaedic Surgeons, New Orleans, 1986.

10. McKee MD, Jupiter JB. Fractures of the distal humerus. Skeletal Trauma Basic Science, Management, and Reconstruction, third edition. Saunders. Philadelphia. 2003:1436-80.

11. Milch H. Fractures and fracture-dislocations of the humeral condyles. J Trauma. 1964;4:592-607.

12. Wickstrom J, Meyer PR. Fractures of the distal humerus in adults. Clin Orthop. 1967:50:43-51.

13. Bryan RS, Morrey BF. Fractures of the distal humerus. In: Morrey, BF, ed. The elbow and its disorders. Philadelphia. W.B. Saunders. 1985;302-39.

14. Jupiter JB, Neff U, Holzach P, et al. Intercondylar fracture of the humerus. J Bone Joint Surg. 1985;67:226-39. 
15. McKee MD, Jupiter JB. A contemporary approach to the management of complex fractures of the distal humerus and their sequelae. Hand Clin. 1994; 10:479-94.

16. McKee MD, Jupiter JB, Toh CL, et al. Reconstruction after malunion and nonunion of intraartiicular fractures of the distal humerus. Methods and results in 13 adults. J Bone Joint Surg Br. 1994;76:614-21.

17. Waddell JP, Hatch J, Richards RR. Supracondylar fractures of the humerus: Results of surgical treatment. J Trauma. 1988;28:1615-21.

18. Henley MB, Bone LB, Parker B. Operative management of intra-articular fractures of the distal humerus. J Orthop Trauma. 1987;1:24-35.

19. Letsch R, Schmit-Neuerburg KP, Sturmer KM, et al. Intraarticular fractures of the distal humerus. Surgical treatment and results. Clin rthop Relat Res. $1989 ; 241: 238-44$

20. Holdsworth BJ, Mossad MM. Fractures of the adult distal humerus. Elbow function after internal fixation. J Bone Joint Surg Br. 1990;72:362-5.

21. Wildburger R, Mahring M, Hofer HP. Supraintercondylar fractures of the distal humerus: results of internal fixation. J Orthop Trauma. 1991;5:301-7.

22. Sodegard J, Sandelin J, Bostman O. Postoperative complications of distal humeral fractures. 27/96 adults followed up for 6 (2-10) years. Acta Orthop Scand. 1992;63:85-9. 
23. Sanchez-Sotelo J, Torchia ME, O’Driscoll SW. Complex distal humeral fractures: Internal fixation with a principle-based parallel-plate technique. J Bone Joint Surg Am. 2007;89:961-9.

24. Ackerman $G$, Jupter, JB. Non-union of fractures of the distal end of the humerus. J Bone Joint Surg Am. 1988;70:75-83.

25. Korner J, Diederichs $G$, Arzdorf M, et al. A biomechanical evaluation of methods of distal humerus fracture fixation using locking compression plates versus conventional reconstruction plates. J Orthop Trauma. $2004 ; 18: 286-93$

26. Ring D, Jupiter JB. Fractures of the distal humerus. Orthop Clin North Am. $2000 ; 31: 103-13$

27. Sanchez-Sotelo J, Torchia ME, O’Driscoll SW. Complex distal humeral fractures: Internal fixation with a principle-based parallel-plate technique. Surgical Technique. J Bone Joint Surg Am. 2008:90S2:31-46.

28. Self J, Viegas SF Jr, Buford WL, et al. A comparison of double-plate fixation methods for complex distal humerus fractures. J Shoulder Elbow Surg. 1995:4:10-6.

29. Jacobson SR, Glisson RR, Urbanaik JR. Comparison of distal humerus fracture fixation: a biomechanical study. J Southern Orthop Assoc. $1997 ; 6: 241-9$ 
30. Schwartz A, Oka R, Odell T, et al. Biomechanical comparison of two perpendicular plating systems for stabilization of complex distal humerus fractures. J Clin Biomech. 2006;21:950-5.

31. Stoffel K, Cunnenn S, Morgan R, et al. Comparative stability of perpendicular versus parallel double-locking plating systems in osteoporotic communited distal humerus fractures. J Orthop Res. 2008;778-84.

32. Schuster I, Korner J, Arzdorf M, et al. Mechanical comparison in cadever specimens of three different 90-degree double-plate osteosyntheses for simulated C2-type distal humerus fractures with varying bone densities. J Orthop Trauma. 2008;22:113-20.

33. Gofton WT, MacDermid JC, Patterson SD, et al. Functional outcome of AO type C distal humeral fractures. J Hand Surg. 2003;28A:294-308.

34. Wong AS, Baratz ME. Elbow Fractures: Distal Humerus. J Hand Surg. 2009:34A: 176-90.

35. Bryan RS, Morrey BF. Extensive posterior exposure of the elbow. A tricepssparing approach. Clin Orthop. 1982;166:188-92.

36. McKee MD, Wilson TL, Winston L, et al. Functional outcome following surgical treatment of intra-articular distal humeral fractures through a posterior approach. J Bone Joint Surg. 2000;82A:1701-7. 
37. McKee MD, Kim J, Kebaish K, et al. Functional outcome after open supracondylar fractures of the huemrus. The effect of the surgical approach. J Bone Joint Surg. 2000;82B:646-51.

38. Knirk JL, Jupiter JB. Intraarticular fractures of the distal end of the radius in young adults. J Bone Joint Surg. 1986;68A:647-59.

39. Athwal GS, Hoxie SC, Rispoli DM, et al. Precontoured parallel plate fixation of AO/OTA type C distal humerus fractures. J Orthop Trauma. $2009 ; 23: 575-80$

40. Ring D, Gulotta L, Jupiter JB. Unstable nonunions of the distal part of the humerus. J Bone Joint Surg. 2003;85A: 1040-6. 


\section{Figure Legends:}

Figure 1: Figure 42-44, from textbook Skeletal Trauma, editors Browner, Jupiter, Trafton, third edition, page \# 1439

Figure 2: Wong AS, Baratz ME. Elbow Fractures: Distal Humerus. J Hand Surg. 2009;34A:176-90. (FIGURE 7)

Figure 3: Sanchez-Sotelo J, Torchia ME, O'Driscoll SW. Complex distal humeral fractures: Internal fixation with a principle-based parallel-plate technique. Surgical Technique. J Bone Joint Surg Am. 2008:90S2:31-46. (FIGURE 1)

Figure 4: Sanchez-Sotelo J, Torchia ME, O'Driscoll SW. Complex distal humeral fractures: Internal fixation with a principle-based parallel-plate technique. Surgical Technique. J Bone Joint Surg Am. 2008:90S2:31-46. (FIGURE 5)

Figure 5: 3D Reconstruction of complex intraarticular distal humerus fracture. The 3D reconstruction demonstrated here can provide additional information on fracture fragment size and orientation in these complex injuries

Figure 6: Anterior 3D Reconstruction of complex intraarticular distal humerus fracture subtracting the radius and ulna. Subtraction of the radius and ulna allows one to fully appreciate the fracture pattern and distinct fracture lines and articular involvement.

Figure 7: Posterior 3D Reconstruction of complex intraarticular distal humerus fracture subtracting the radius and ulna. The 3D construct can be rotated real time in many planes to allow a thorough understanding of the fracture.

Figure 8: Intraoperative View of ORIF of this fracture using a 90-90 plating technique. Note the placement of the plates and the preliminary K-wires which are placed to aid 
in the reduction and allow easier plate application. Precontoured plates can make plate application easier, but some contouring may still be necessary.

Figures 9, 10: Intraoperative view demonstrating final placement of the plates applied using a 90-90 plating technique. Note the excellent reduction achieved as well as the excellent visualization allowed using an olecranon osteotomy.

Figures 11,12: Postoperative AP and Lateral Radiographs demonstrating anatomic reduction with union using a $90-90$ plating technique. Note the fixation of the olecranon osteotomy using a precontoured olecranon plate.

Figure 13, 14: Preoperative AP and Lateral Radiographs demonstrating a complex intraarticular fracture of the distal humerus.

Figure 15: Intraoperative view of this complex distal humerus fracture. The vessel loop is protecting the ulnar nerve which should be mobilized routinely in all of these complex cases. An olecranon osteotomy has been performed to aid in fracture visualization.

Figure 16, 17: Postoperative AP and Lateral Radiographs demonstrating anatomic reduction and union of this complex distal humerus fracture using a parallel plating technique. Note the number of screws that can be placed in the distal fragments. In addition, the olecranon osteotomy has been fixed using a double wire tension band technique. 